\title{
Core Loss in Fe-Si Powder Cores
}

\author{
T. Saito and S. Takemoto* \\ Daido Corporate Research \& Development Center, Daido Steel Co., Ltd., 2-30 Daido-cho, Minami-ku, Nagoya 457-8545, Japan \\ * Metal Powder Div., Daido Steel Co., Ltd., 10 Ryugu-cho, Minato-ku, Nagoya 455-0022, Japan
}

The applications of powder cores to very high power conversion equipment have recently been spreading. Fe-Si alloys are effective in downsizing magnetic cores because they have large degrees of magnetization. However, core loss needs to be further reduced. The relationships between core losses for Fe-3mass\%Si powder cores made under various conditions were investigated in this study, i.e., hysteresis losses, classical eddy current losses, and anomalous eddy current losses including the sizes of their particles and crystal grains. We found that hysteresis losses decreased as crystal grains increased in size. We confirmed that not only there were classical eddy current losses generated uniformly in particles, but also anomalous eddy current losses occurred. The anomalous eddy current losses suggested that they were due to domain wall movement, and they decreased with decreasing crystal grain sizes. Their exponents of the frequency dependency of anomalous eddy current losses varied between 1.5 and 2.0 depending on test conditions, and they reflected the change in the number of domains with frequency.

Key words: powder core, core loss, hysteresis loss, classical eddy current loss, anomalous eddy current loss

\section{Introduction}

Soft magnetic compressed powder cores that are made of surface insulated soft magnetic powder have been widely used for the choke coils of switched mode power supplies and DC-DC converters. Furthermore, their use has recently spread to high power conversion equipment such as the inverters of hybrid vehicles and photovoltaic generators by replacing conventional $\mathrm{Si}$ steel sheets.

As Fe-3mass\%Si alloy has high saturation magnetization, it is suitable for miniaturizing magnetic cores. However, their core loss is comparatively larger than that in conventional powder cores of $\mathrm{Fe}-\mathrm{Si}-\mathrm{Al}$ (Sendust) and Mo-permalloy, and therefore, lower loss is required from the point of view of lower heat generation and higher efficiency. Moreover, core loss must be reduced significantly for higher driving frequencies in the near future.

The authors previously reported the influences of particle size, crystal grain size, and core resistivity on the hysteresis losses and eddy current losses of $\mathrm{Fe}-\mathrm{Si}$ powder cores ${ }^{1), 2}$. This paper mainly describes particle and crystal grain sizes, and the frequency dependencies of eddy current losses including those from the previous results.

\section{Experimental procedure}

Fe-3mass\%Si alloy powders were prepared by using a method of modified water-atomization. The powders were sieved to make various particle distributions of -150 $\mathrm{\mu m} /+106 \mu \mathrm{m}$ (mean diameter $=\sim 128 \mu \mathrm{m}$ ), -75 $\mu \mathrm{m} /+63 \mu \mathrm{m}$ (mean diameter $=\sim 69 \mu \mathrm{m}),-63 \mu \mathrm{m} /+45 \mu \mathrm{m}$ (mean diameter $=\sim 54 \mu \mathrm{m})$, and $-45 \mu \mathrm{m}$ (mean diameter $=\sim 23 \mu \mathrm{m}$ ). Figure 1 has a representative SEM image for a $-75 \mu \mathrm{m} /+63 \mu \mathrm{m}$ particle. These particles were almost

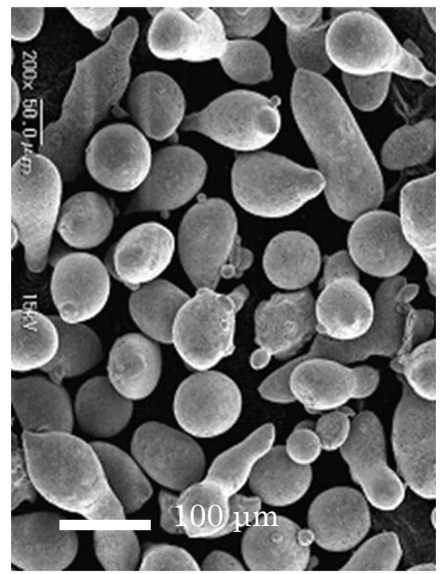

Fig. 1 SEM image of tested powder $(-75 \mu \mathrm{m} /+63 \mu \mathrm{m})$

round or spherical for every size distribution.

The powders were heat treated at 948 and $1223 \mathrm{~K}$ and held at $10.8 \mathrm{ks}$ under a hydrogen gas atmosphere. The maximum temperature of $1223 \mathrm{~K}$ was chosen in order to prevent the particles from cohering. Then, silicone resin binder was added at 0.5 mass $\%$ to insulate particles from other particles. Toroidal cores with an outer diameter of $28 \mathrm{~mm}$, an inner diameter of $20 \mathrm{~mm}$, and a height of approximately $5 \mathrm{~mm}$ were made by press forming under a pressure of $1960 \mathrm{MPa}$ at room temperature (RT). Finally, the cores were heat treated at 948 and $1023 \mathrm{~K}$ and held at $3.6 \mathrm{ks}$ in an $\mathrm{Ar}$ gas atmosphere combined with powder heat treatment to change the sizes of the crystal grains. The maximum temperature of $1023 \mathrm{~K}$ was chosen to insulate particles from other particles. Core losses were measured at various frequencies with a fixed exciting magnetic flux density of $0.1 \mathrm{~T}$. The measured core losses were separated into hysteresis losses and eddy current losses. The losses per cycle were extrapolated to zero hertz, and the intersection was defined as the hysteresis loss per 
cycle, which was similar to the method described in the previous report ${ }^{1}$. The remnant obtained by subtracting the hysteresis loss from core loss was considered to be eddy current loss.

About 100 crystal gain sizes, which were observed in the cross section of each core with an optical microscope, were measured and then averaged in order to evaluate the crystal grain sizes in the particles.

\section{Results and discussions}

\subsection{Change in loss with particle size}

Figures 2 (a) and (b) plot the dependencies of core losses, hysteresis losses, and eddy current losses on particle size at $10 \mathrm{kHz}$ and $0.1 \mathrm{~T}$, and at $100 \mathrm{kHz}$ and 0.1 $\mathrm{T}$, respectively. The hysteresis losses for both frequencies decrease with increased particle size; however, the eddy current losses increase with increased particle size. The core loss at $10 \mathrm{kHz}$ is minimum around $50 \mu \mathrm{m}$ as a result of the balance between hysteresis loss and eddy current loss. The eddy current losses at 100
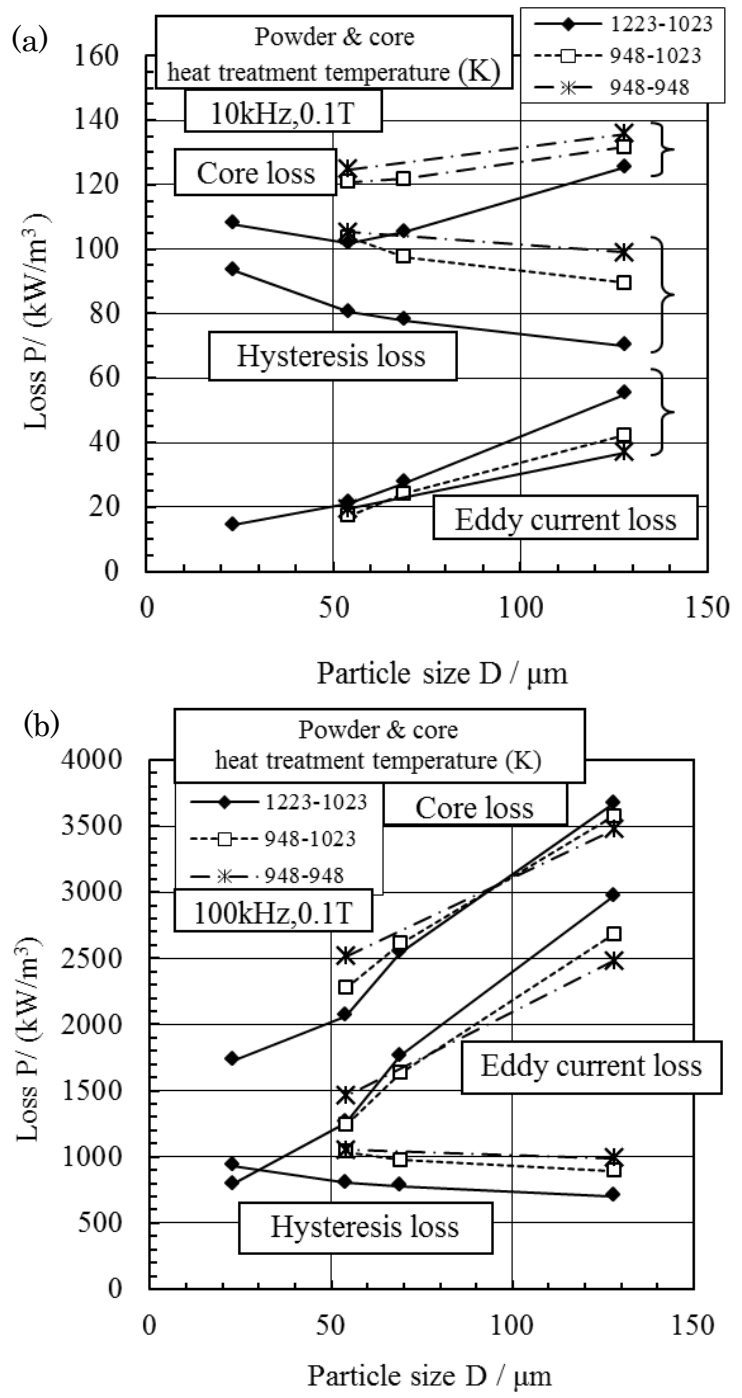

Fig. 2 Changes of core loss, hysteresis loss and eddy current loss at (a) $10 \mathrm{kHz}, 0.1 \mathrm{~T}$ and (b) $100 \mathrm{kHz}, 0.1 \mathrm{~T}$ with particle size.
$\mathrm{kHz}$ are more dominant than hysteresis losses, and core losses therefore tend to increase as the particle size increases.

The heat treatment conditions resulted in some differences in losses. The hysteresis losses were the lowest for the heat treatments with the highest temperature, i.e., the powder heat treatment temperature of $1223 \mathrm{~K}$ and the core heat treatment temperature of $1023 \mathrm{~K}$. The eddy current losses were the lowest for the lowest temperature, i.e., the powder heat treatment temperature of $948 \mathrm{~K}$ and the core heat treatment temperature of $948 \mathrm{~K}$, except for part of the data.

\subsection{Change in crystal grain size}

Figure 3 presents the microstructures of heat-treated crystal grain sizes under two typical conditions. It is can be seen that grain sizes become coarser at high temperature heat treatments.

Figure 4 shows the changes in crystal grain sizes with particle sizes. The crystal grain sizes increase with

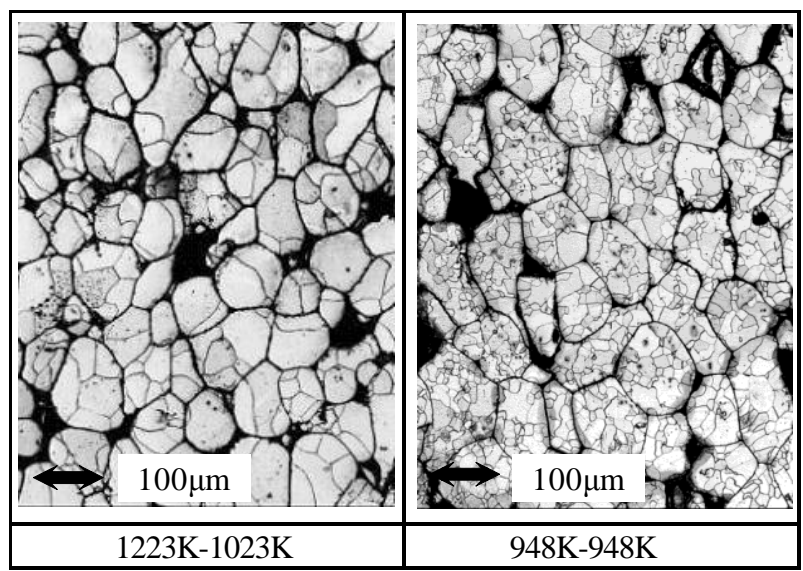

Fig. 3 Microstructures of tested cores with -150 $\mu \mathrm{m} /+106 \mu \mathrm{m}$ particle size. Powder-core heat treatment temperatures are indicated.

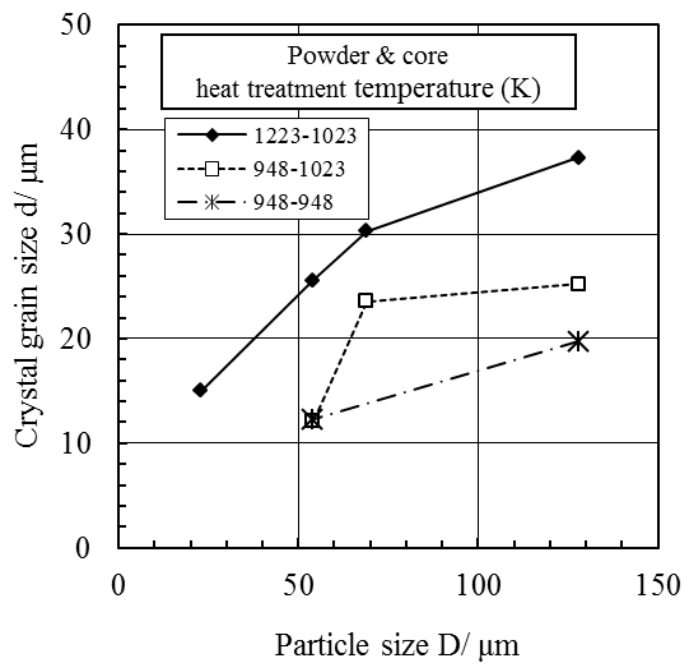

Fig. 4 Changes of crystal grain size with particle size. 
the increase in particle sizes, and they are also larger under heat treatment conditions at high temperatures.

\subsection{Generation factors in core loss}

It is well known that core loss is composed of hysteresis loss, which is static loss and eddy current loss, which is a kind of dynamic loss in metal powder cores. Moreover, eddy current loss is supposed to generally consist of three types of modes. Figure 5 shows a schematic of the mechanism of eddy current loss generation. One is inter-particle eddy current that flows between neighboring particles, and the other is intra-particle eddy current that flows in each particle. Furthermore, the intra-particle eddy current involves two factors. One is eddy current that flows uniformly in particles, which is classified as classical eddy current loss. The other is microscopic eddy current loss that is generated around a magnetic domain due to movement of the domain wall. This microscopic eddy current loss is called anomalous eddy current loss or excess loss, and the above phenomena and mechanism have been clarified for conventional Si steel sheets ${ }^{3)-5}$. Consequently, the total loss is expressed as the sum of several losses:

$$
P_{c}=P_{h}+P_{e(\text { inter })}+P_{e(c l)}+P_{e(a n)},
$$

where $P_{c}$ is core loss, $P_{h}$ is hysteresis loss, and $P_{e(i n t e r)}$ is eddy current loss due to inter-particle eddy current. Here, $P_{e(c l)}$ is eddy current loss due to intra-particle uniform eddy current (classical eddy current loss) and $P_{e(a n)}$ is eddy current loss due to domain wall movement (anomalous eddy current loss).

Moreover, there may also be loss due to rotation magnetization, but this generally appears at higher frequencies such as those above the megahertz range ${ }^{6)}$. Hence, the influences on loss for frequencies of less than $100 \mathrm{kHz}$ in this study are assumed to be small. The $P_{\text {e(inter) }}$ in Eq. (1) can also be neglected. As has previously been reported ${ }^{2)}$, as the resistivity of all tested cores was over $0.1 \Omega \mathrm{m}$, it is therefore thought that the insulation between neighboring particles is sufficiently maintained

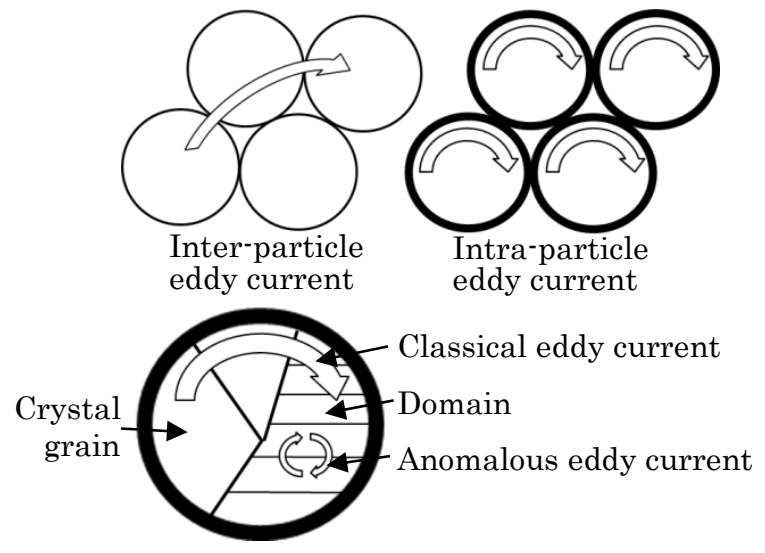

Fig. 5 Mechanism of eddy current generation. so that eddy current only flows inside particles. The $P_{h}$, $P_{e(c l)}$, and $P_{e(a n)}$ are analyzed in the subsections that follow.

\subsection{Change in hysteresis loss with crystal grain size}

Figure 6 plots the relationship between hysteresis loss at $100 \mathrm{kHz}$ and $0.1 \mathrm{~T}$ and crystal grain size. Hysteresis losses decrease with larger crystal grain sizes for all particle sizes. This tendency is similar to that of general bulk soft magnetic materials.

\subsection{Change in classical eddy current loss and anomalous eddy current loss with particle and crystal grain sizes}

Classical eddy current loss in powder cores for spherical magnetic particles can be calculated ${ }^{7)}$ as:

$$
P_{e(c l)}=\frac{\left(\pi B_{m} f D\right)^{2}}{20 \rho_{(\text {particle })} \eta},
$$

where $P_{e(c l)}$ is classical eddy current loss $\left[\mathrm{W} / \mathrm{m}^{3}\right], \eta$ is the volume content of magnetic particles, and $B_{m}$ is excited magnetic flux density [T]. Here, $f$ is frequency $[\mathrm{Hz}], D$ is particle size $[\mathrm{m}]$, and $\rho_{\text {(particle) }}$ is the resistivity of particles $[\Omega \mathrm{m}]$.

Here, $\eta$ was around 0.92 , and $\rho$ (particle) was $45 \times 10^{-8}$ which was the same as that of the bulk.

$P_{h}$ was separated from the measured $P_{c}$, and then the $P_{e(c l)}$ calculated with Eq. (2) was subtracted, leaving $P_{e(a n)}$. Figures 7 (a) and (b) plot the dependencies of $P_{e(c l)}$ and $P_{\text {e(an })}$ on particle size at $10 \mathrm{kHz}$ and $0.1 \mathrm{~T}$, and 100 $\mathrm{kHz}$ and $0.1 \mathrm{~T}$, respectively. As can be understood from Eq. (2), $P_{e(c l)}$ increases in proportion to the square of particle size. However, observed eddy current loss cannot only be explained by $P_{e(c l)}$, and the contribution of $P_{e(a n)}$ is confirmed. We also found that eddy current losses are large for coarser crystal grain sizes.

It is known that $P_{e(a n)}$ is related to domain width and is proportional to the square root of crystal grain size ${ }^{3), 8)}$ as:

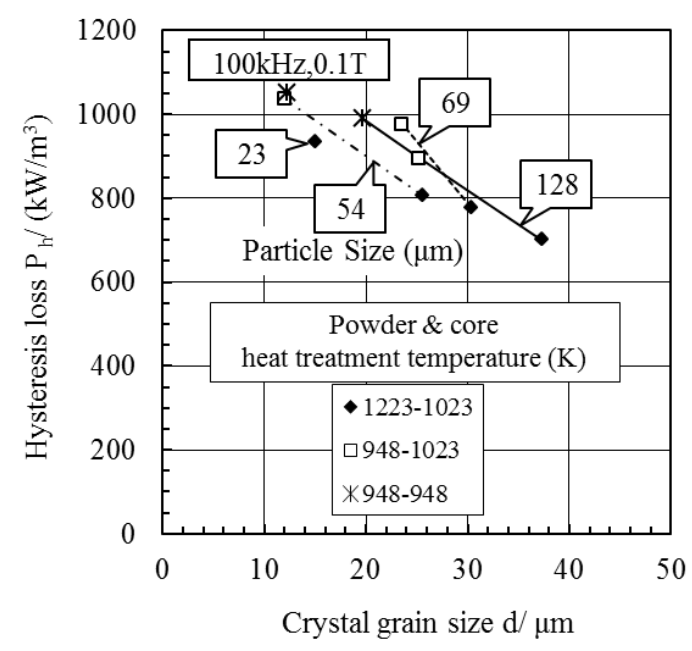

Fig. 6 Relationship between hysteresis loss and crystal grain size. 


$$
P_{e(a n)}=\alpha w=\alpha^{\prime} \sqrt{d},
$$

where $w$ is domain width [m] and $d$ is the crystal grain size $[\mathrm{m}]$.

Then, using separated $P_{e(a n)}$, coefficient $\alpha$ ' was determined by using the mean of the least squares method, and the following predictions were obtained for $10 \mathrm{kHz}$ and $0.1 \mathrm{~T}$ and $100 \mathrm{kHz}$ and $0.1 \mathrm{~T}$.

$$
\begin{array}{lll}
P_{e(a n)}=4.30 \times 10^{6} \sqrt{d} & (10 \mathrm{kHz}, 0.1 \mathrm{~T}) & (4) \quad \text { and } \\
P_{e(a n)}=1.91 \times 10^{8} \sqrt{d} & (100 \mathrm{kHz}, 0.1 \mathrm{~T}) & (5)
\end{array}
$$

The sums of $P_{e(c l)}$ and $P_{e(a n)}$ calculated with Eqs. (2), (4), and (5) are indicated in Figs. 7 by the dotted lines for several crystal grain sizes. We found that the contribution of $P_{e(a n)}$ in eddy current loss was so significant that it was necessary to optimally control the crystal grain size to reduce core loss.

Figure 8 plots the changes in eddy current loss $\left(P_{e(c) l}+P_{e(a n)}\right)$ at $100 \mathrm{kHz}$ and $0.1 \mathrm{~T}$ with changes in crystal grain size. Tendencies for eddy current losses to increase with increased crystal grain size can be seen.
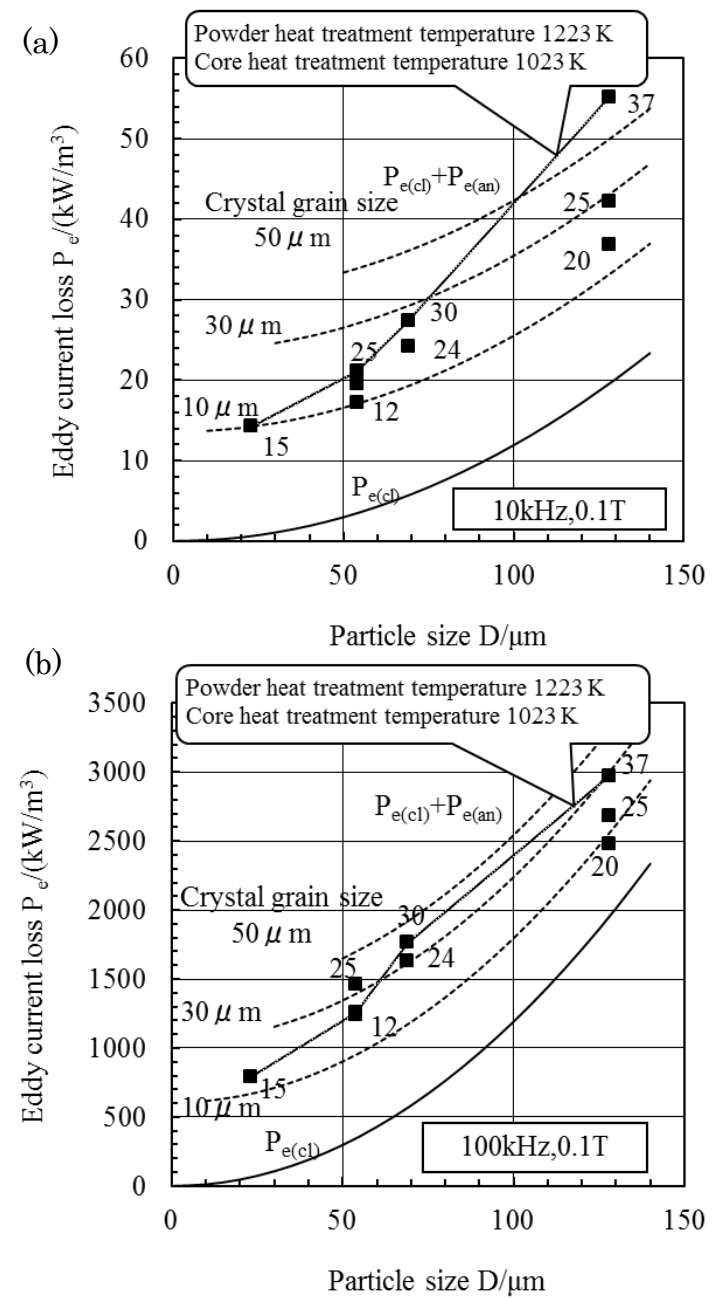

Fig. 7 Relationship between eddy current loss at (a) 10 $\mathrm{kHz}, 0.1 \mathrm{~T}$, (b) $100 \mathrm{kHz}, 0.1 \mathrm{~T}$ and particle size. The solid line and broken lines show calculated results with Eqs. (2), (4) and (5). The numbers inside the figures are measured crystal grain size.

\subsection{Frequency dependence of anomalous eddy current loss}

This section discusses the dependencies of anomalous eddy current losses on frequency. Figure 9

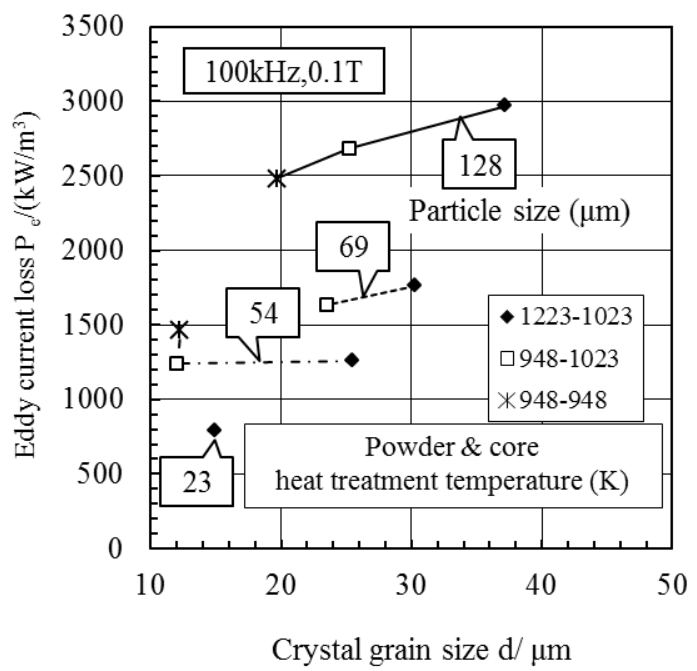

Fig. 8 Relationship between eddy current loss and crystal grain size.

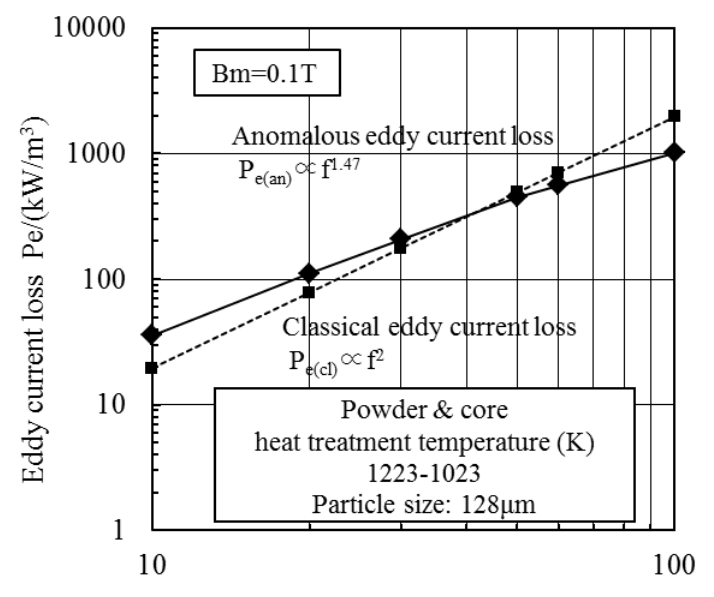

Freqency f/kHz

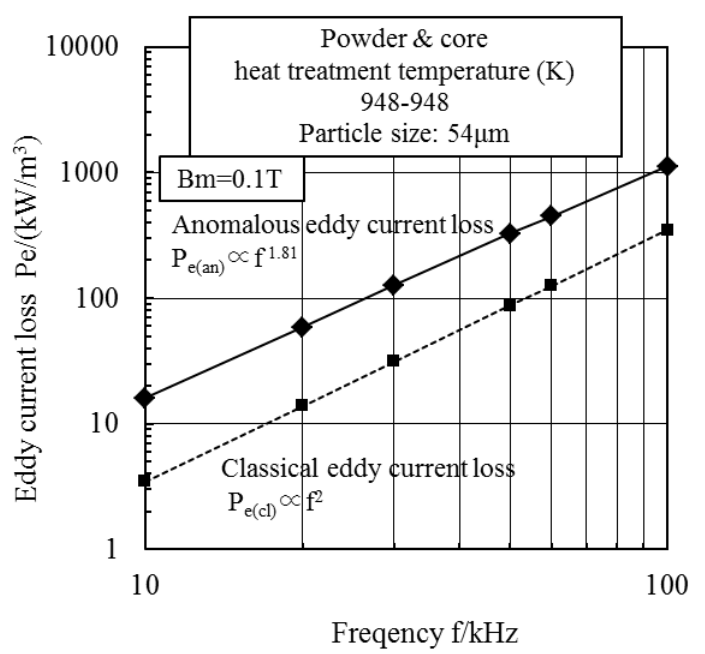

Fig. 9 Frequency dependencies of eddy current loss. 


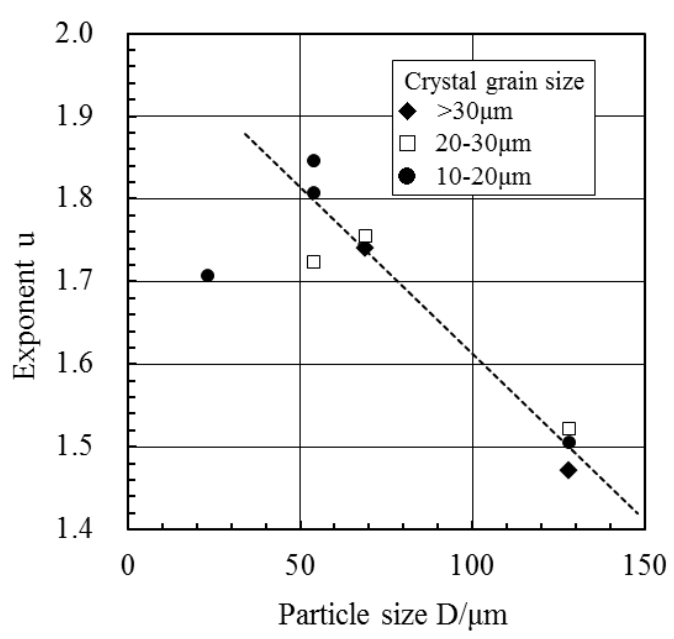

Fig. 10 Relationship between particle size and exponent of frequency dependency of anomalous eddy current loss.

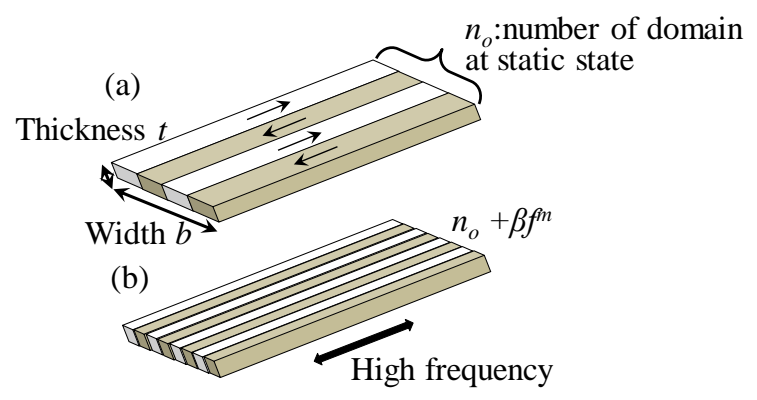

Fig. 11 Assumed schematic domain model.

presents frequency dependencies of $P_{e(c l)}$ and $P_{e(a n)}$ for two examples under different test conditions. $P_{e(c l)}$ was calculated with Eq. (2) and is proportional to the square of frequency as understood from this equation. However, the exponent of the dependence of $P_{e(a n)}$ on frequency is less than 2, and this is different to that under test conditions. The relationship between particle size and the exponent for the frequency dependence of $P_{e(a n)}$ under all tested conditions is plotted in Fig. 10. The exponent varies between around 1.5 and 2.0. It also tends to decrease with increased particle size, and it seems to have a smaller exponent for coarser crystal grain sizes.

The frequency dependence of $P_{e(a n)}$ is discussed relative to the frequency dependence of the number of domains below. Based on the proposal by Sakaki et al., anomalous eddy current loss in a divided simple bar like that in the $180^{\circ}$ domain is given by Eqs. (6) and (7) ${ }^{5}$, which have been slightly modified from the original.

$$
\begin{gathered}
P_{e(a n)}=\frac{8.4 b t B_{m}{ }^{2} f^{2}}{\pi n \rho} \quad \text { (6) and } \\
n=n_{0}+\beta f^{m}(m \doteqdot 0.5), \quad \text { (7) }
\end{gathered}
$$

where $b$ is the width including that of divided domains [m], $t$ is the thickness of domains [m], and $n$ is the number of domains included in width $b$ in the dynamic state. Here, $n_{0}$ is the number of domains included in width $b$ in the static state and $\beta$ is the coefficient.

The schematic domain model assumed in the above equations is illustrated in Fig. 11 where (a) indicates the situation in the static state, and (b) indicates that in the dynamic state with narrower domains. We predicted that the domain configurations would be more complex for powder cores, but the fundamental frequency dependence of $P_{e(a n)}$ must be expressed with Eqs. (6) and (7).

Sakaki et al. describe that the number of domains increases with about the $0.5^{\text {th }}$ power of frequency from the experimental results on several ribbon shaped soft magnetic materials, e.g., amorphous and permalloy. Moreover, Mohri et al. computed the dynamic domain wall energy for an Si steel sheet and obtained results where the number of domains increases with about the $0.4^{\text {th }}$ power of frequency ${ }^{4}$.

Sakaki et al. assumed $n \doteqdot \beta f^{m}$, but considering the number of domains in static state $n_{o}$ here, the frequency dependencies of $P_{\text {e(an) }}$ are discussed using Eqs. (6) and (7).

Two extreme conditions can be assumed; first, when $n_{0}$ is much smaller than $\beta f^{m}, P_{e(a n)}$ is proportional to the $1.5^{\text {th }}$ power of frequency. In the opposite situation, when $\beta f^{n}$ is much smaller than $n_{0}, P_{e(a n)}$ is proportional to the square of frequency. Therefore, depending on the magnitude relation of $n_{0}$ and $\beta f^{m}$, the exponent of the frequency dependence of $P_{e(a n)}$ varies from 1.5 to 2.0 on average. Speculating from the above discussion, we find that the change in the exponent with particle size in Fig. 10 suggests the magnitude relation of $n_{0}$ and $\beta f^{m}$. However, as the domain structures of the samples tested in this study are not clear, further detailed discussions would not have any significant meaning. It is necessary to observe the domain configurations in detail and to clarify the relationships between them and the anomalous eddy current losses, as well as to investigate theoretical approaches.

\section{Conclusions}

The authors investigated what influences particle and crystal grain sizes had on core losses, i.e., hysteresis losses and eddy current losses for $\mathrm{Fe}-3$ mass\%Si powder cores made with various particle sizes and under different heat treatment conditions. Four main conclusions were drawn.

1) Hysteresis losses decreased as crystal grain size increased, which was achieved by high temperature heat treatment.

2) Classical eddy current losses were generated uniformly in particles, and anomalous losses were also found. This suggested that magnetic domain wall movements caused the anomalous losses, which is a phenomenon that is similar to that reported for conventional Si steel sheets.

3) Anomalous eddy current losses decreased as the crystal grain size became fine. 
4) The exponent of the frequency dependencies of anomalous eddy current losses changed between 1.5 and 2.0, and it tended to approach 1.5 as particle size increased. The exponents of 1.5-2.0 suggested that the number of domains increased with frequency.

\section{References}

1) T. Saito and S. Takemoto: Electric Furnace Steel, 74, 285 (2006).

2) T. Saito, S. Takemoto and T.Iriyama: IEEE on Trans. on Magn., MAG-41, 3301 (2005).

3) R. H. Pry and C. P. Bean: J. Appl. Phys. 29, 532 (1958)
4) K. Mohri, Y. Sato and T. Fujimoto: The Transactions of the Institute of Electrical Engineers of Japan, A17, 125 (1977).

5) Y. Sakaki and S. Imagi: The Transactions of the Institute of Electrical Engineers of Japan, A73, 575 (1981).

6) S. Chikazumi: Kyoujiseitai no Butsuri 2 (in Japanese), p. 319 (Syokabo, Tokyo, 1984).

7) K. Ohta: Jikikogaku no Kiso 2 (in Japanese), p. 311 (Kyoritsu Shuppan, Tokyo, 1973).

8) S. Chikazumi: Kyoujiseitai no Butsuri 2 (in Japanese), p. 200 (Syokabo, Tokyo, 1984).

Received Oct. 17, 2012; Revised Nov. 27, 2012; Accepted Dec. 13, 2012 